\title{
SITUACIÓN DEL SARAMPIÓN EN ESPAÑA. ESTUDIO SEROEPIDEMIOLÓGICO
}

\section{Isabel Pachón del Amo.}

Centro Nacional de Epidemiología. Instituto de Salud Carlos III. Ministerio de Sanidad y Consumo.

El sarampión figura como enfermedad de declaración obligatoria en nuestro país desde el año 1900, mediante una circular de la Dirección General de Sanidad concretada al año siguiente, en la cual se establece la obligatoriedad, para todos los médicos, de declarar los casos de una lista de once enfermedades infecto-contagiosas ${ }^{1}$.

En la actualidad, el Sistema de Enfermedades de Declaración Obligatoria (EDO) requiere la notificación, a nivel estatal, de todo caso sospechoso de sarampión de forma numérica, con periodicidad semanal y ámbito geográfico provincial y a nivel de comunidad autónoma.

A partir de 1997, con la entrada en vigor de la Red Nacional de Vigilancia Epidemiológica (RNVE) según Real Decreto 2210/1995 del 28-XII, se requiere el envío de un informe anual, en el que consten todos los casos notificados de forma numérica durante el año precedente con las características individuales de edad, sexo, estado de vacunación, tipo de caso, semana de notificación y ámbito geográfico.

En los protocolos de las enfermedades de declaración obligatoria de la $\mathrm{RNVE}^{2}$, se aplica, para el sarampión, la siguiente definición de caso:

Caso sospechoso/probable: Todo aquél que cumple los criterios de definición clínica de caso, caracterizado por rash generali- zado de duración ${ }^{3} 3$ días, con fiebre ${ }^{3} 38,5^{\circ} \mathrm{C}$ $\mathrm{y}$ tos o coriza o conjuntivitis. No tiene confirmación virológica o serológica y no está cpidemiológicamente relacionado con otro caso sospechoso o confirmado.

Caso confirmado: Todo caso sospechoso que es confirmado por el laboratorio mediante aislamiento del virus del sarampión de especímenes clínicos o aumento significativo en el nivel de anticuerpos frente al virus del sarampión, mediante un método serológico estándar o test positivo serológico de anticuerpos IgM de sarampión. Así mismo, se define como caso confirmado todo caso sospechoso que concuerda con la definición clínica de caso y está epidemiológicamente relacionado con uno confirmado o con otro caso sospechoso.

Una actividad muy importante del sistema de vigilancia epidemiológica del sarampión hace referencia a la notificación de brotes, así como su inmediata investigación y aplicación de medidas de control.

En la serie temporal anual de casos notificados de sarampión en España (figura 1), se observa un primer período de una alta incidencia con cifras medias anuales de 429 por 100.000 (150.000 casos), y la presencia de ciclos bianuales característicos de esta infección. Este período abarca la época prevacunal y los primeros años tras la administración de la vacuna. 
Figura 1

Sarampión en España: 1940-1997. Incidencia anual y cobertura de vacunación

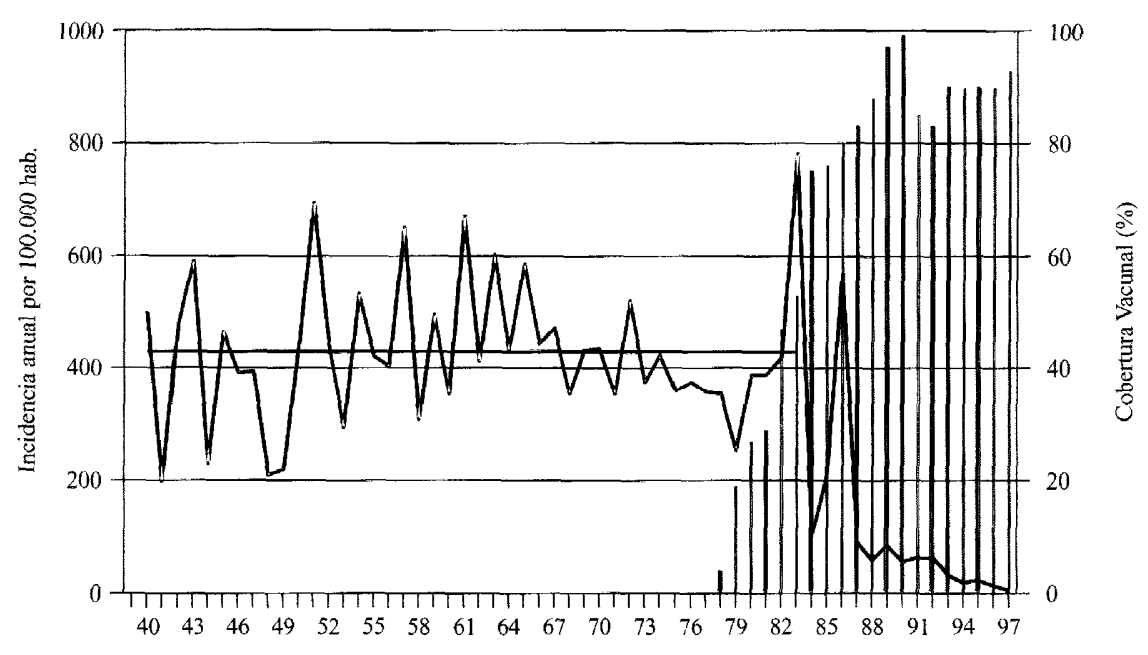

En 1978, empieza a aplicarse en nuestro país la vacuna frente al sarampión, aumentando su cobertura de forma muy lenta y paulatina, no alcanzando altas coberturas, próximas al $80 \%$, hasta el año 1987. Durante ese período se detectan dos importantes picos epidémicos, en los años 1982 y 1986.

A partir de 1987 se produce un descenso muy importante en la incidencia de la enfermedad y entre 1987 y 1993 se observa una incidencia media anual de 42 casos por 100.000 habitantes $(25.000$ casos). Esta disminución continúa detectándose en los últimos años, alcanzando una cifra de 4,7 por 100.000 en 1997 ( 1.838 casos) y hasta octubre de 1998 se ha detectado una incidencia de 1,02 por 100.000 con 392 casos notificados (figura 2).

Por lo que se refiere a la distribución geográfica, la disminución de la incidencia anual es prácticamente generalizada en todas las provincias, como se puede obscrvar en ambos mapas. En 1997, solo una provincia no detectó ningún caso de sarampión y 14 pro- vincias notificaron una incidencia anual ${ }^{2} 1$ por 100.000 . Hasta octubre de 1998, ocho provincias no han detectado ningún caso $\mathrm{y}$ 26 provincias han notificado una incidencia anual ${ }^{2} 1$ por 100.000 , cifra que se considera óptima en el control del sarampión. En este último año solo Las Palmas y Ceuta han notificado una incidencia $>5$ por 100.000 .

La información individualizada obtenida de los casos notificados en 1997 permite conocer determinadas características de los mismos (figura 3). Un 28,1\% de los casos han sido confirmados, si bien se desconoce el criterio seguido. El $64,9 \%$ consta como caso sospechoso, el $28,1 \%$ como caso confirmado y en un $7,0 \%$ se desconoce el tipo de caso. El 54,9\% ocurren en varones. El $26,5 \%$ refiere estar vacunado si bien hay un alto porcentaje $(40,3 \%)$ en el que se desconoce esta información.

En cuanto a la edad de presentación de los casos, cabe destacar que el $8,6 \%$ se presenta en menores de 1 año y que más del $50 \%$ se da en el rango de edad entre los 10-19 años. 
Figura 2

Sarampión en España: 1987-1998*. Incidencia anual

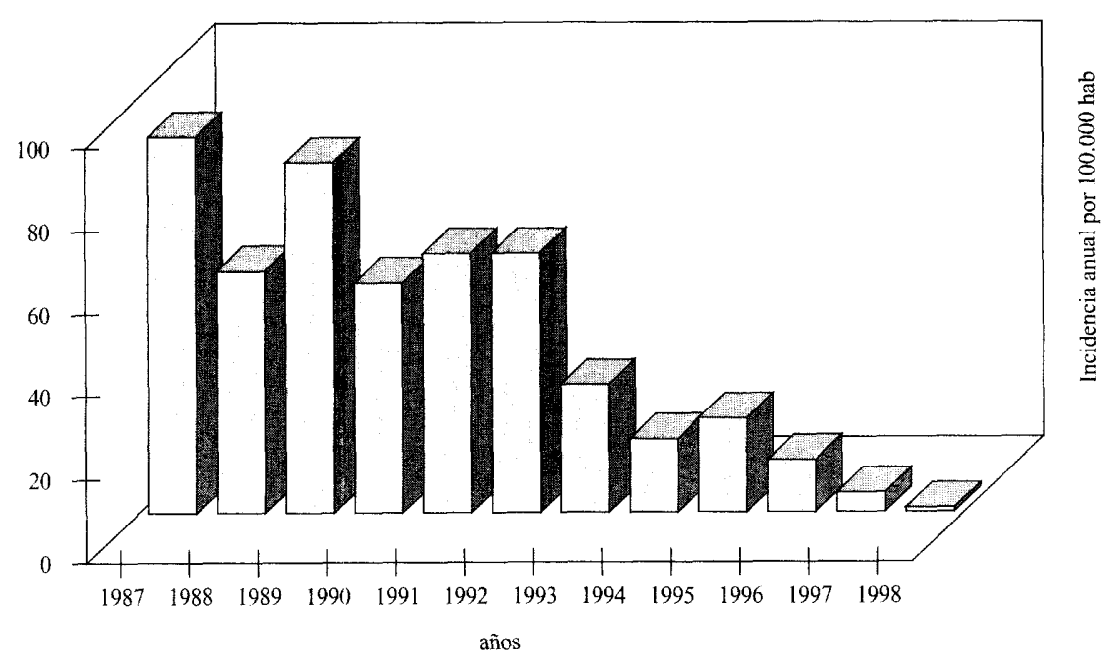

Figura 3

Sarampión por provincia en España. Incidencia anual

AÑO 1997

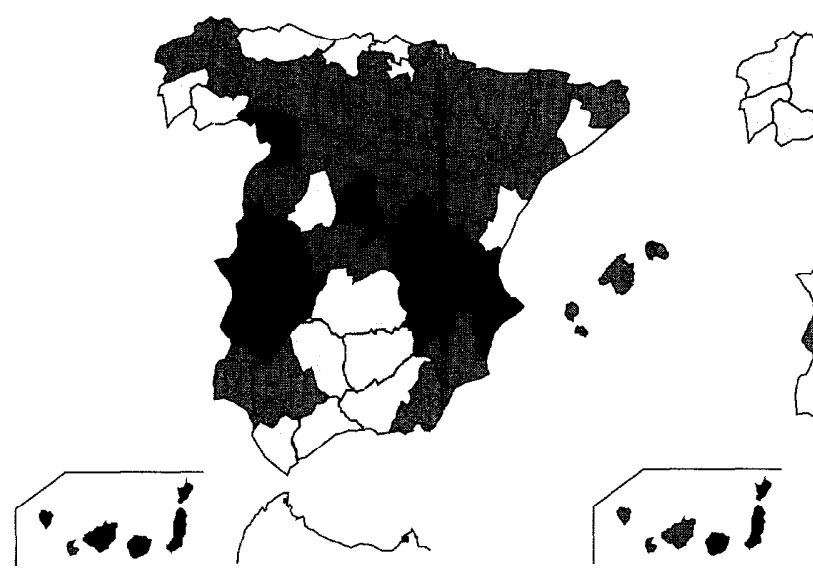

AÑO 1998*

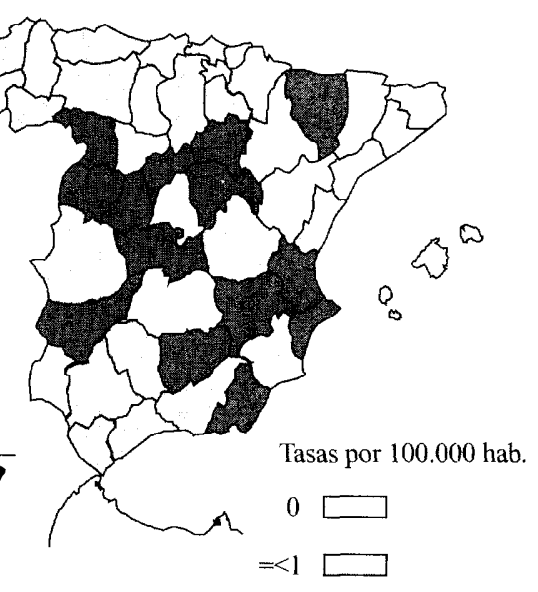

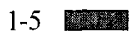

$>5$ 
El $83,3 \%$ de los casos se presentan en sujetos menores de 20 años.

Se ha recogido información de hospitalización a través del Conjunto Mínimo Básico de Datos (CMBD) correspondiente al año 1996. Esta base de datos registra los diagnósticos hospitalarios al alta y se estima que, para el año 1996, tenía una cobertura hospitalaria del 95\%. El número de casos hospitalizados por sarampión ha sido de 168 de los cuales el $82,6 \%$ figura sin complica- ciones y un 7,2\% con complicaciones no especificadas; las complicaciones presentadas en el resto de los casos han sido: queratitis $(1,2 \%)$, neumonía $(6,0 \%)$ y encefalitis $(3,0 \%)$. Un $96,4 \%$ de los casos ha sido dado de alta por curación y se ha registrado un caso $(0,6 \%)$ de defunción (figura 4$)$. En cuanto a la edad de presentación de los casos hospitalizados es muy similar a la descrita en la información individualizada de casos notificados, presentándose un 86,3\% en menores de 20 años (figura 5).

\section{Figura 4}

Sarampión año 1997. Información individualizada

Tipo de caso

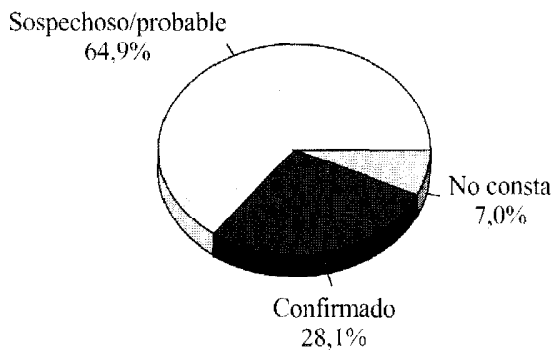

Vacunación

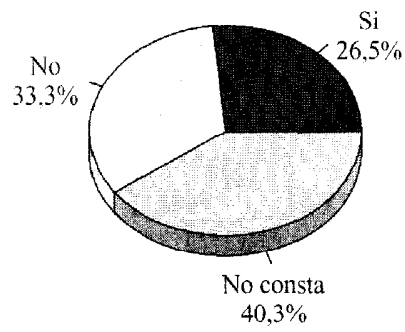

En la figura 6 se presenta la mortalidad en España por grupos de edad, recogida a través del Instituto Nacional de Estadística. Se puede observar la casi desaparición de la misma en los últimos tres años, con solo un caso de defunción registrado, si bien, habría que chequear las fuentes de información de mortalidad de forma muy precisa para con-
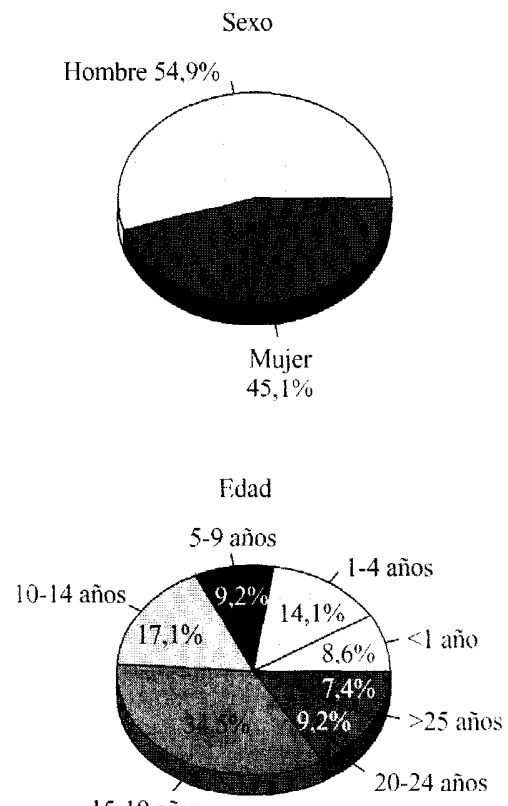

15-19 años firmar la posible existencia de defunciones por sarampión.

En 1996 se realizó una encucsta de scroprevalencia con representatividad nacional, excepto Cataluña, cuyo objetivo fundamental era conocer el estado de inmunidad de la población frente a determinados agentes in- 
Figura 5

Hospitalización. Conjunto Mínimo Básico de Datos: 1996
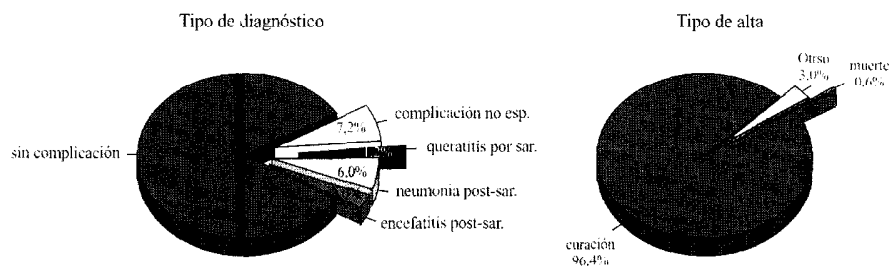

N." de casos hospitalizados: 168 .

Figura 6

Hospitalización. Conjunto Mínimo Básico de Datos: 1996. Casos por edad

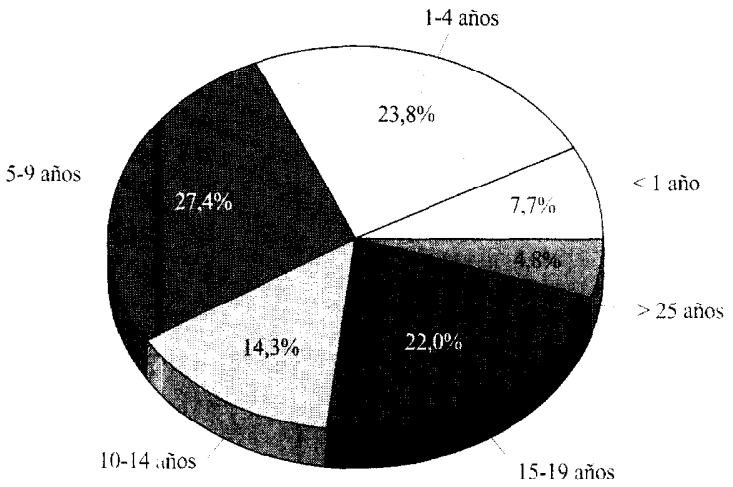

fecciosos, entre los que figuraba el virus del sarampión. Para ello se realizó un muestreo polietápico por conglomerados, considerando como conglomerado a los centros de extracción de sangre periférica de la red de asistencia pública sanitaria. Se tuvieron en cuenta dos estratos, rural y urbano, y siete grupos de población comprendida entre los 2 y 39 años. El tamaño total de muestra obtenido fue de 3.932 sujetos, 2.085 en el medio urbano y 1.847 en el medio rural.

A partir del estudio de seroprevalencia se calculó la cobertura de vacunación en los niños de 2 a 12 años para cada una de las vacunas que se administran en el programa de va- cunación infantil, previa solicitud de la cartilla de vacunación para obtener la información. Como se observa en la figura 7 , el $96,1 \%$ de los niños de $2-5$ años tienen el esquema básico completo de vacunación que consta de, al menos, tres dosis de vacuna oral frente a la poliomielitis, tres dosis de vacuna triple bacteriana (Difteria-Tétanos- Pertussis) y una dosis de vacuna triple virica (Sarampión-Rubéola- Parotiditis). En el grupo de 6 a 9 años, el $94,6 \%$ de los niños han recibido dicho esquema básico completo.

La seroprevalencia de anticuerpos detectables frente a sarampión fue superior al $95 \%$ en todos los grupos de edad excepto en 
Figura 7

Sarampión en España: 1980-1995. Mortalidad por grupo de edad

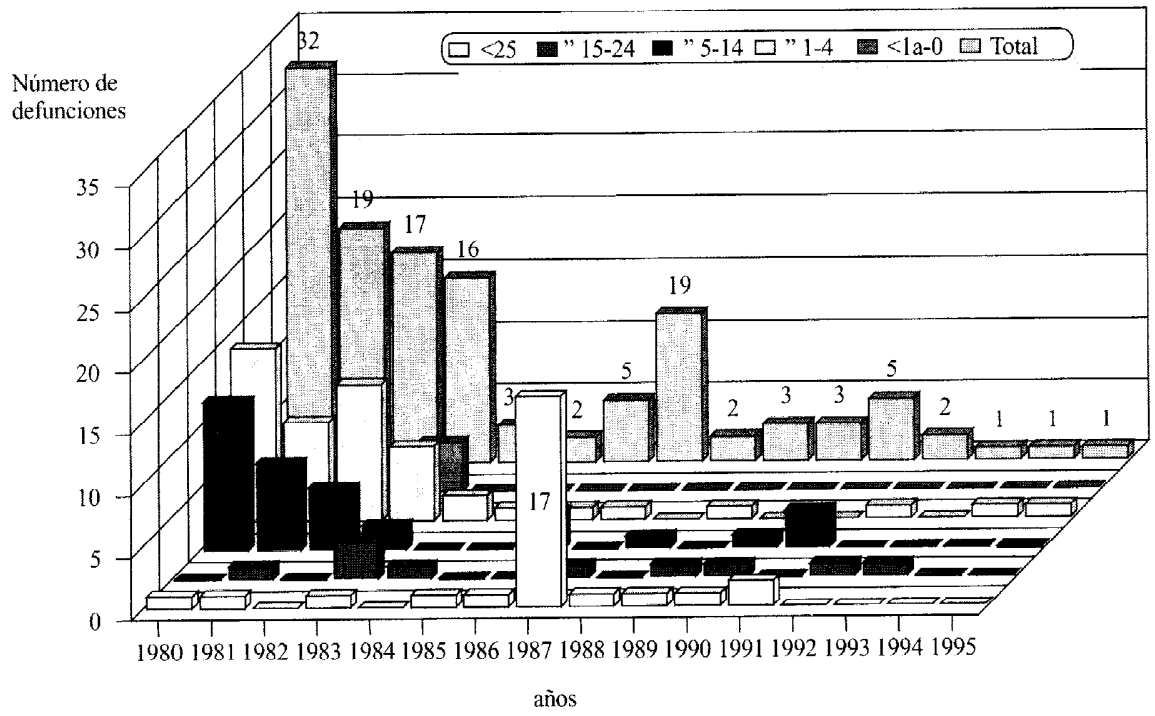

Fuente: Instituto Nacional Estadistica

el de 6 a 9 años, en el que fue del $90,8 \%$, y en el de 15 a 19 años que fue del $94,5 \%$; a partir de los 20 años la prevalencia es superior al 98\% (figura 8 ).

Figura 8

Encuesta Nacional de seroprevalencia, 1996. Cobertura del programa de vacunación. Esquema básico completo
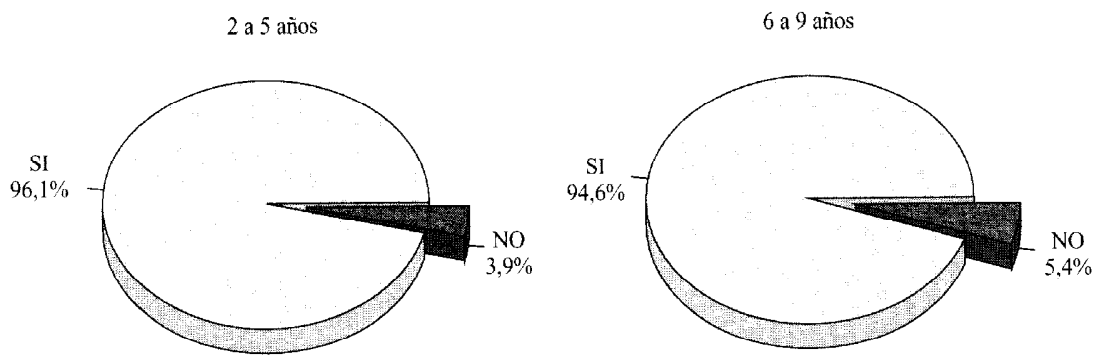
La mayor proporción de población susceptible se encuentra, pues, en el grupo de 6-9 años, con un 9,2\%, como se puede observar en la gráfica 9. Únicamente el grupo de 15 a 19 años presenta niveles de susceptibilidad ligeramente superiores a los reco- mendados por la Organización Mundial de la Salud (OMS) en sus estrategias de eliminación del sarampión. En la figura que representa el acúmulo de población susceptible se observa que, hasta la edad de 20 años, se acumula el $80 \%$ de todos los susceptibles.

Figura 9

Sarampión. Población inmune por edad. Encuesta nacional de seroprevalencia, 1996

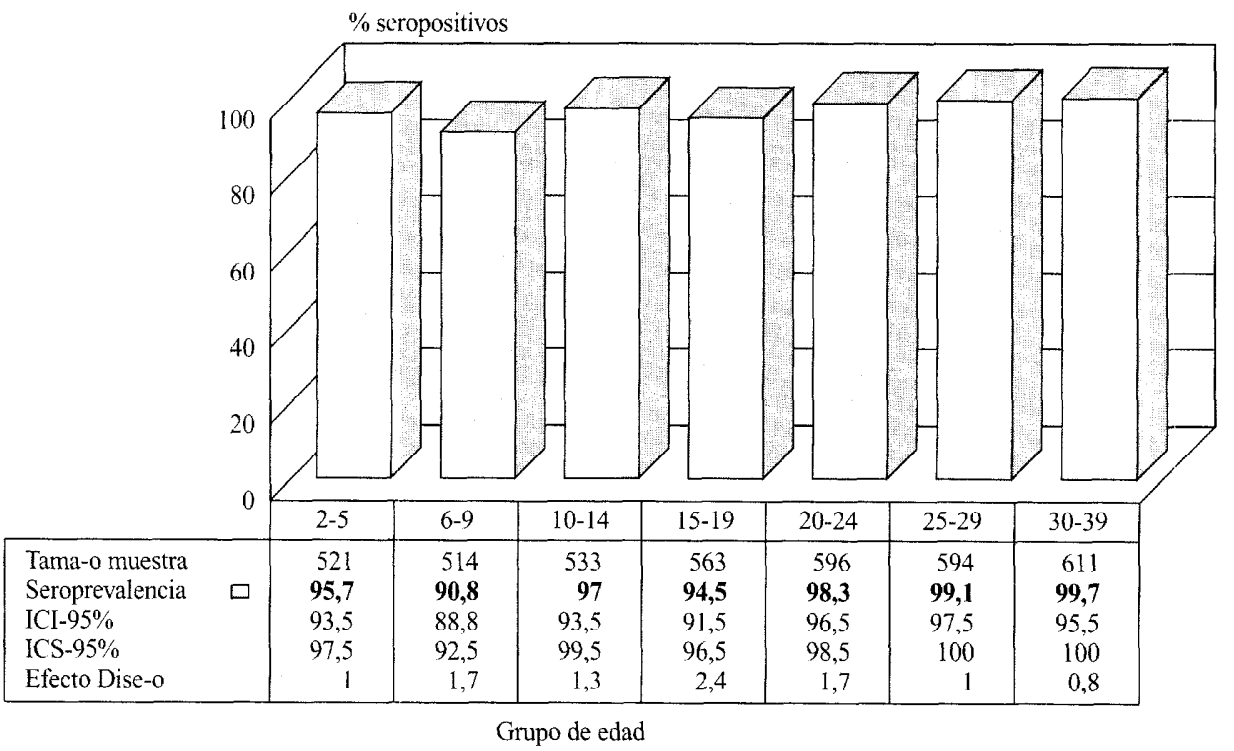

La importante disminución de la incidencia de sarampión detectada desde la introducción de la vacunación y el logro de unas altas coberturas en el momento actual, indican la posibilidad de alcanzar la eliminación del sarampión en nuestro país. El logro de dicho objetivo se posibilitaría con la aplicación de algunas de las estrategias recomendadas por la $\mathrm{OMS}^{3-4}$, tales como:

- Alcanzar y mantener una alta cobertura en los programas de inmunización infantil, asegurando que las mismas se alcanzan en todas las unidades geográ- ficas y estudiar la necesidad o no de adoptar alguna estrategia específica que acelere el proceso de eliminación del sarampión, tales como campañas de catch-up o mopping-up.

- Mejorar y reforzar el sistema de vigilancia epidemiológica del sarampión potenciando la capacidad de: a) detectar de forma inmediata la circulación del virus del sarampión, b) investigación a fondo de los brotes para indagar los factores de riesgo de sarampión, c) identificar áreas o subpoblaciones en 
las que pueda estar ocurriendo fallos del programa de vacunación. Es imprescindible un análisis pormenorizado de los datos de vigilancia, incluyendo la investigación de brotes, para tomar decisiones acerca de las medidas de control y ajustar las estrategias en el programa de climinación de la enfermedad.

- Importancia del papel de los laboratorios en el programa de eliminación. A medida que la incidencia de la enfermedad desciende a niveles muy bajos, se hace necesaria la confirmación por laboratorio de todos los casos sospechosos de sarampión, por lo que será preciso el establecimiento de una red de laboratorios y la estandarización de los criterios de diagnóstico ${ }^{5}$.

La vigilancia virológica, incluyendo el aislamicnto e identificación del genotipo del virus circulante, es un factor muy importante en la vigilancia rutinaria y para evaluar los progresos hacia la eliminación del sarampión.

\section{BIBLIOGRAFÍA}

1. Martinez Navarro F. Algunos problemas en la reconstrucción de las series históricas de las estadísticas demográfico-sanitarias. V Encuentro Marcelino Pascua: Estadisticas demográfico-sanitarias. Madrid: Ministerio de Sanidad y Consumo. Instituto de Salud Carlos III; 1991.

2. Centro Nacional de Epidemiología. Protocolos de enfermedades de declaración obligatoria. Madrid. Ministerio de Sanidad y Consumo; 1996.

3. Ramsay M. Strategic Plan for the elimination of measles in the European Region. Expanded Programme on Immunization. Seventh meeting of national programme managers. Alemania: 1997.

4. CDC. Advances in Global Measles Control and Elimination: Summary of the 1997 International Mceting. MMWR 1998; 47 (N." RR-11).

5. Canada Communicable Disease Report. Measles surveillance:guidelines for laboratory support. CCDR 1998: 24-5: 33-44. 